\title{
Computational model for long-range non-linear propagation over urban cities
}

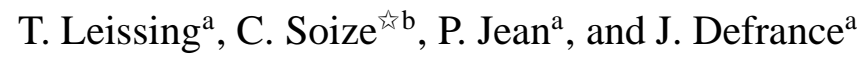 \\ ${ }^{a}$ Université Paris-Est, Centre Scientifique et Technique du Bâtiment, 24 rue Joseph Fourier, 38400 Saint Martin \\ d'Hères, France \\ ${ }^{b}$ Université Paris-Est, Laboratoire Modélisation et Simulation Multi Echelle, MSME FRE3160 CNRS, 5 bd \\ Descartes, 77454 Marne-la-Vallee, France
}

\begin{abstract}
A computational model for long-range non-linear sound propagation over urban environments is described. First the probability model of the geometrical parameters of an urban environment are determined using Information Theory and the Maximum Entropy Principle. The propagation model is then presented: it is based on the non-linear parabolic equation (NPE) and its extension to propagation in porous media, in which the urban layer of the real system is represented by a porous ground layer. The uncertainties introduced by the use of this simplified model and the presence of the variability of the real system are taken into account with a probabilistic model. Reference solutions are obtained thanks to the boundary element method (BEM); these experimental observations are then used to identify the parameters of the probability model. This inverse stochastic problem is solved using an evolutionary algorithm which involves both the mean-square method and the maximum likelihood method. Applications and model validation are then presented for two different urban environment morphologies. It is shown that the identification method provides an accurate and robust way for identifying the stochastic model parameters, independently of the variability of the real system. Constructed confidence regions are in good agreement with the numerical observations.
\end{abstract}

Key words: long-range propagation, urban environment, parabolic equation, maximum entropy principle, evolutionary algorithm, maximum likelihood

\footnotetext{
${ }^{2}$ Corresponding author

Email addresses: thomas. leissing@cstb.fr (T. Leissing), christian.soize@univ-mlv.fr (C. Soize $\left.{ }^{\text {tr }}\right)$ Preprint submitted to Elsevier 


\section{Introduction}

The long-term aim of this work is to develop a computational model to simulate long-range non-linear wave propagation over urban cities. We are interested in the effect of the surface irregularities (buildings) on the acoustic field above the urban layer. This problem could be studied with deterministic numerical models where buildings geometries are explicitly given. For example, ray tracing methods or models based on the Euler's equations allow the environmental context (buildings, meteorological conditions, site topography, ...) to be taken into account and would technically be suited for this application. However, for long-range propagation applications, these methods suffer from their numerical complexity and the high computational effort associated. Moreover, the suitability of these models for the application under interest can be questioned: considering the high complexity of the real system, the model approximations (e.g. using the uniform theory of diffraction for ray-tracing methods) and the uncertainties on the model parameters (e.g. buildings geometries), the computational model could be improved introducing a probabilistic model.

In this work, a different approach is proposed. It consists in using a very simple model for the urban and atmospheric layers. Propagation is modelled with a non-linear parabolic equation (NPE), whose fundamental principle is the solution of a one-way non-linear wave equation over a moving window surrounding the wavefront. Since the calculation domain is limited to a small area around the signal, computational cost is generally reduced compared to Euler's equations methods. For the propagation in the urban layer, a NPE for porous ground layers is used. Therefore the simplified model is composed of two domains:

(1) the atmospheric layer, where propagation is modelled by a NPE for air,

(2) the urban city layer, where propagation is modelled by a NPE for porous ground surface.

Equations to couple the two domains complete the simplified model of sound propagation over urban cities. Using this model as a predictive model for the real system will show very poor performances as the modelling chosen is far too simplistic to represent the real system complexity. Hence a probabilistic approach of uncertainties is used to enhance the model capabilities. Section 1.1 below describes the scope and the range of applicability of such a propagation model. 


\subsection{Scope and range of applicability}

The work presented in this paper was performed to study high-amplitude wave propagation in complex media [22]. The sound source of such a wave could for example be a sonic bang or the wave produced by a ground-level explosion. High-amplitude effects are hence considered and the frequency range under interest is restricted to $0-100 \mathrm{~Hz}$.

It is not the intent here to develop an engineering model for the calculation of noise exposure in urban areas (such as the Nord 2000 model, see [20], or the Harmonoise model, see [41, 11]), but rather to present a method for the construction of a stochastic model for propagation over irregular surfaces, such as an urban environment. The propagation over the urban environment, and not within, is hence considered in this paper.

The urban environment is further characterised by a set of three random variables: the heights and widths of the buildings, and the spacing between two consecutive buildings (see section 3 for a description of these parameters). It is not the intent here to determine what the statistical dependencies between these parameters are. The probability distributions of these three random variables are determined using Information Theory [33] and the maximum entropy principle [17].

It was chosen to place the source above the urban city layer (see section 4 for a complete description of the configuration): this could correspond to the case of the propagation of a sonic bang. As a description of the sound field above the city layer is sought, the receiver is placed at the same altitude as the source. Note that nothing prevents one to use the presented method for source and receiver locations at any other location, or for a wider frequency range.

As explained in section 1 a NPE model is used as a basis for the development of the stochastic propagation model. This choice was made regarding the application under interest in this work. The NPE model is well adapted for long-range sound propagation applications and it can account for most features of weakly non-linear sound propagation outdoors: geometrical spreading, weak non-linearities, refraction effects (see for example [27, 6]), site topography [24], ground impedance [23] and thermoviscous effects [39]. Different models, such as the Fast Field Program (FFP, see [14, 31]) or the (linear, frequency-domain) Parabolic Equation (PE, see [15]) could as well be used. The method for the construction of the stochastic model presented in this paper is general and is not restricted to the use of a specific propagation model. 
Section 2 explains and details the methodology to construct such a stochastic sound propagation model and the probabilistic model of its parameters, and outlines the content of the paper.

\section{Construction of the computational model: principles and methodology}

The objective is to develop a stochastic model for sound propagation over urban cities using a NPE model originally designed for sound propagation over porous ground layers, in which the urban city is taken into account through independent random porous layer parameters, noted $\Gamma$, $\Lambda$ and $\Theta$. The probability distributions of these random variables depend on a parameter vector $\mathbf{w}$ (bold letters denote vectors throughout the paper). For example parameter $\mathbf{w}$ may contain the mean values and the standard deviations associated with the random parameters $\Gamma, \Lambda$ and $\Theta$. The model output is the pressure at the receiver which is noted $P_{r}(\omega)$. A second propagation model, in which the urban city is explicitly accounted for, is used to provide reference solutions. The urban city geometry is characterised by a parameter vector $\mathbf{u}$. For example, $\mathbf{u}$ may contain the mean values and standard deviations associated with the heights and widths of the buildings (parameter $\mathbf{u}$ is defined in section 3 ). For a given parameter $\mathbf{u}$ and several probability models an urban city realisation can be generated, and then used in the reference model to obtain the pressure $P_{r}^{\exp }$ at the receiver. Figure 1 shows a sketch and a diagram that detail the basic principle of each model.

To construct the stochastic sound propagation model, for a given parameter $\mathbf{u}$, one has: (1) to construct the probability models of the urban city geometrical parameters. From one city to another one, geometrical parameters (e.g. building density, mean elevation, ... ) can greatly vary. The construction of a probabilistic model of these parameters should hence be done with measured data (for example, data from a geographical information system). Moreover, town planning and buildings themselves complies with many constraints which introduce a statistical dependence between the geometrical parameters. The determination of these dependencies being out of the scope of this work, probability models are constructed assuming that no information is available concerning the relations between geometrical parameters. The probability models are determined with the help of Information Theory [33] and the Maximum Entropy Principle [17]. Once the probability models of the geometrical parameters are determined, different city realisations corresponding to a given parameter $\mathbf{u}$ can be generated. 
(2) to use the city realisations generated in (1) and to perform simulations with the reference model in order to obtain statistical information on the model output $P_{r}^{\exp }(\omega)$.

(3) to construct the probability models of the random parameters $\Gamma, \Lambda$ and $\Theta$. These models are determined with the help of Information Theory and the Maximum Entropy Principle and depend on parameter $\mathbf{w}$.

(4) using the outputs from the reference model $P_{r}^{\exp }(\omega)$ (step (2)), to identify parameter $\mathbf{w}$ corresponding to the given parameter $\mathbf{u}$ previously fixed. This identification is done by solving an inverse stochastic problem: the "distance" between $P_{r}(\omega ; \mathbf{w})$ and $P_{r}^{\exp }(\omega)$ is minimised so that the optimal parameter $\mathbf{w}^{\text {opt }}$ is obtained. Once $\mathbf{w}^{\text {opt }}$ is determined, the stochastic NPE model can be used to study non-linear wave propagation over urban cities.

The paper is organised as follows. Section 3 presents the method to construct the probability models of an urban city (step (1)). The linear reference propagation model used to obtain reference solutions (step (2)) is detailed in section 4 while the mean NPE model for sound propagation over urban cities and its parameters are presented in section 5. In section 6 , the probability models of the NPE model random variables are determined (step (3)). The stochastic NPE model is then presented in section 7. Section 8 deals with a hybrid method based on the mean-square and the maximum likelihood methods to solve the inverse stochastic problem and to identify the parameter $\mathbf{w}^{\text {opt }}$ (step (4)). Finally, section 9 presents an application and a validation procedure for the computational model of sound propagation over urban cities. Conclusions and perspectives are given in section 10 .

\section{Prior probabilistic model of geometrical parameters of an urban city}

A two dimensional cross-section of an urban city is considered. It is composed of $n$ buildings of rectangular shape parametrised by a set of three parameters. For a given building $B_{i}$, its height and width are noted $h_{i}$ and $w_{i}$, and the distance between two consecutive buildings $B_{i}$ and $B_{i+1}$ is noted $d_{i}$ (see figure 2). The prior probability model of such an urban city is then introduced with the help of the random variables $\left\{H_{1}, \ldots, H_{n}\right\},\left\{W_{1}, \ldots, W_{n}\right\},\left\{D_{1}, \ldots, D_{n-1}\right\}$. Let $X_{i}$ be the positive-valued real random variable representing either $H_{i}$, either $W_{i}$, or $D_{i}$. Let $p_{X_{i}}$ be the probability density function of $X_{i}$ and $E$ be the mathematical expectation. Let $m_{X_{i}}$ and $\delta_{X_{i}}=\sigma_{X_{i}} / m_{X_{i}}$ be its mean value 

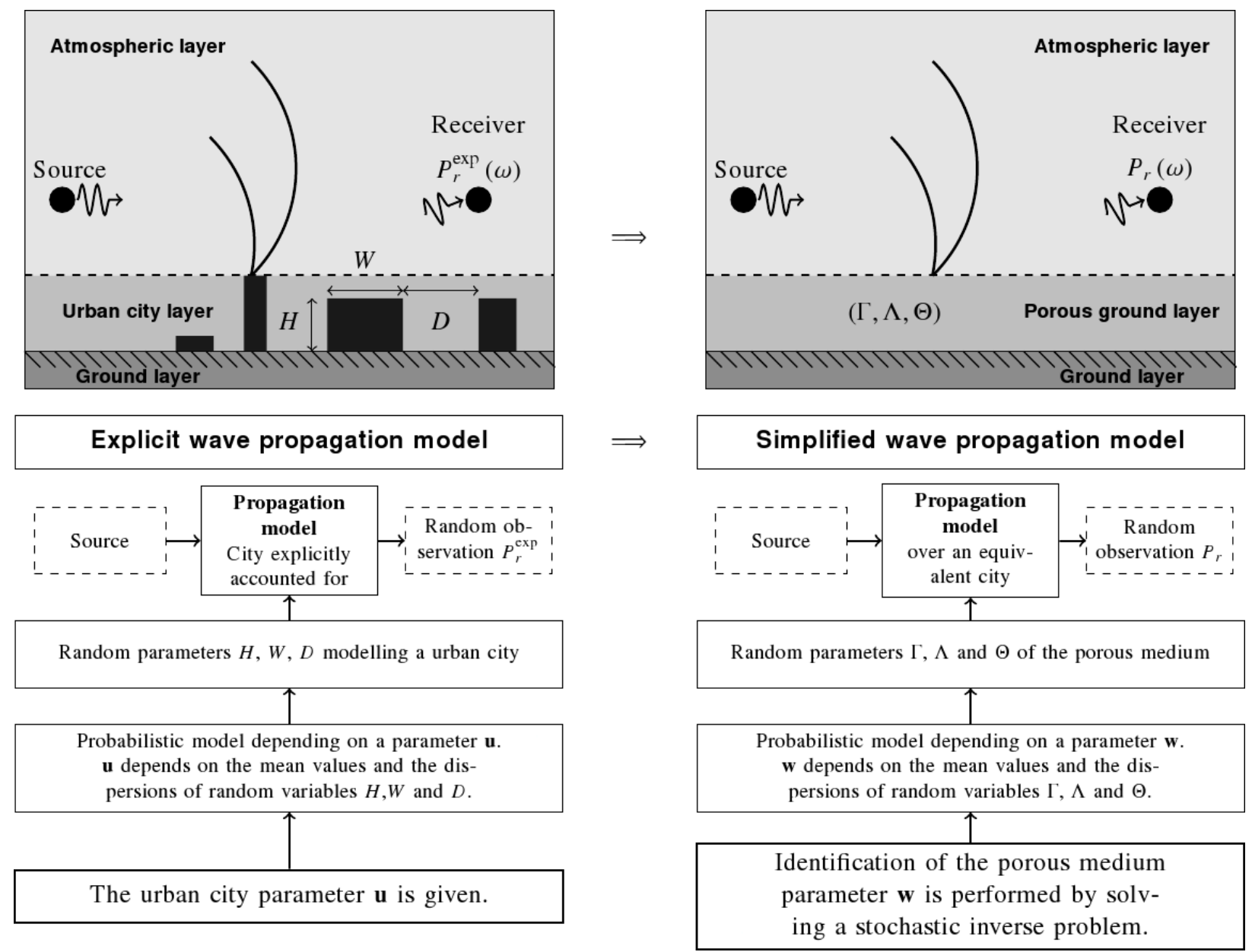

Figure 1: Sketches and diagrams detailing the basic principles of each model. The reference, linear propagation model is presented on the left, while the stochastic simplified NPE model is shown on the right.

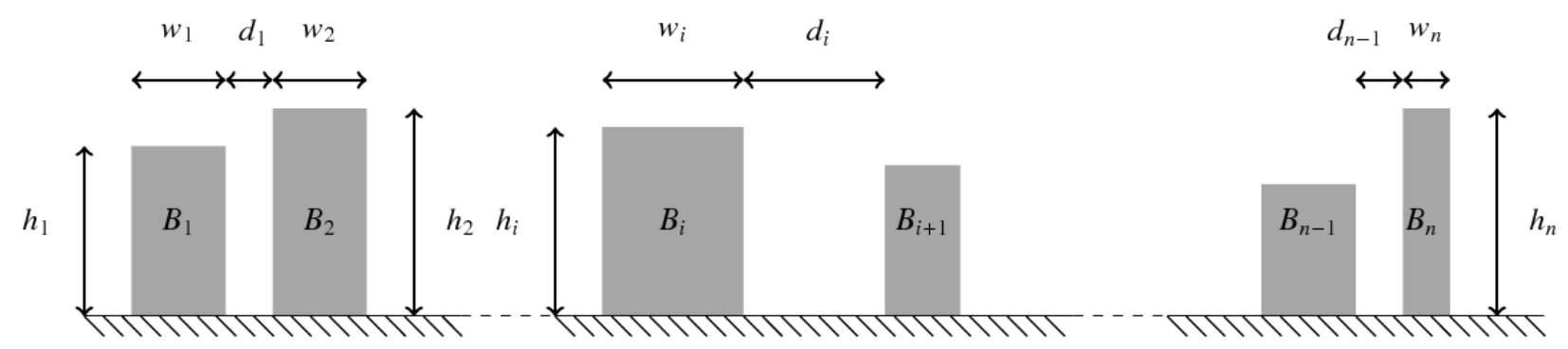

Figure 2: Sketch of a representative urban city. 
and its coefficient of variation, in which $\sigma_{X_{i}}^{2}=m_{2}-m_{X_{i}}^{2}$ is the variance and $m_{2}$ the second-order moment. One has:

$$
\begin{aligned}
& m_{X_{i}}=E\left\{X_{i}\right\}=\int_{0}^{+\infty} x_{i} p_{X_{i}} d x_{i}, \\
& m_{2}=E\left\{X_{i}^{2}\right\}=\int_{0}^{+\infty} x_{i}^{2} p_{X_{i}} d x_{i} .
\end{aligned}
$$

In order to construct the probability distributions of random variables $\left\{H_{1}, \ldots, H_{n}\right\},\left\{W_{1}, \ldots, W_{n}\right\}$, $\left\{D_{1}, \ldots, D_{n-1}\right\}$ Information Theory [33] and the Maximum Entropy Principle [17] are used. One then has to define the available information for these random variables, which is the following: For all $i$, denoting by $X_{i}$ either $H_{i}$, either $W_{i}$ or $D_{i}$ one has:

(1) $X_{i}$ is a random variable with values in $] 0,+\infty[$

(2) The mean value $m_{X_{i}}=E\left\{X_{i}\right\}$ is given and is equal to $m_{X}$, independent of $i$

(3) The inverse $X_{i}^{-1}$ of $X_{i}$ is a second-order random variable. This condition is satisfied if

$$
E\left\{\log \left(X_{i}\right)\right\}=c_{i}, \quad \text { with }\left|c_{i}\right|<+\infty
$$

The constraint defined by equation (2) introduces an arbitrary constant $c_{i}$ which does not have any physical meaning and which is then rewritten as a function of the coefficient of variation of $X_{i}$ which is $\delta_{X}$, independent of $i$. The use of the Maximum Entropy Principle yields [34]:

$$
\begin{gathered}
p_{H_{1}, \ldots, H_{n}, W_{1}, \ldots, W_{n}, D_{1}, \ldots, D_{n-1}}\left(h_{1}, \ldots, h_{n}, w_{1}, \ldots, w_{n}, d_{1}, \ldots, d_{n-1}\right)= \\
\prod_{i=1}^{n} p_{H_{i}}\left(h_{i}\right) \prod_{i=1}^{n} p_{W_{i}}\left(W_{i}\right) \prod_{i=1}^{n-1} p_{D_{i}}\left(d_{i}\right),
\end{gathered}
$$

with

$$
\begin{aligned}
& p_{H_{i}}\left(h_{i}\right)=p_{X}\left(h_{i} ; m_{H}, \delta_{H}\right), \\
& p_{W_{i}}\left(w_{i}\right)=p_{X}\left(w_{i} ; m_{W}, \delta_{W}\right), \\
& p_{D_{i}}\left(d_{i}\right)=p_{X}\left(d_{i} ; m_{D}, \delta_{D}\right)
\end{aligned}
$$

where

$$
p_{X}\left(x ; m_{X}, \delta_{X}\right)=\mathbb{1}_{] 0,+\infty[}(x) \frac{1}{m_{X}}\left(\frac{1}{\delta_{X}^{2}}\right) \frac{1}{\Gamma\left(1 / \delta_{X}^{2}\right)}\left(\frac{x}{m_{X}}\right)^{\frac{1}{\delta_{X}^{2}}-1} \exp \left(-\frac{x}{\delta_{X}^{2} m_{X}}\right)
$$


In equation $(5), \mathbb{1}_{] 0,+\infty[}(x)=1$ if $x>0$ and 0 otherwise, and $\Gamma(z)$ is the gamma function of argument $z$ defined by:

$$
\Gamma(z)=\int_{0}^{+\infty} t^{z-1} e^{-t} d t
$$

Since no available information concerning the statistical dependence between the families of random variables $\left\{H_{i}\right\}_{i},\left\{W_{i}\right\}_{i}$ and $\left\{D_{i}\right\}_{i}$ is used, the Maximum Entropy Principle yields independence of all the random variables as a result, as it can be seen in equation (3).

The following vector $\mathbf{u}$ of the parameters of the probabilistic model is introduced:

$$
\mathbf{u}=\left(m_{H}, m_{W}, m_{D}, \delta_{H}, \delta_{W}, \delta_{D}\right)
$$

Parameter $\mathbf{u}$ hence contains the mean values and the dispersion parameters associated with the heights, widths and spacings of the buildings. It belongs to an admissible set $\mathcal{U}=(] 0,+\infty[)^{6}$. Figure 3 shows an example of a realisation with $n=20$ and $\mathbf{u}=(10,20,30,0.2,0.2,0.2)$.

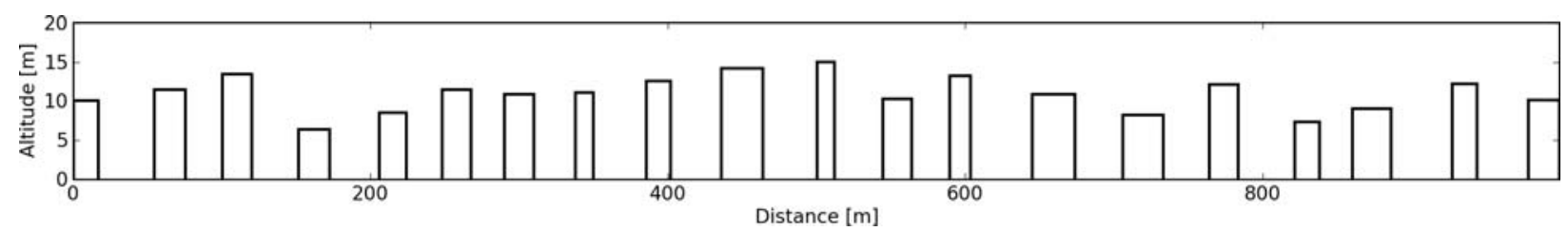

Figure 3: Example of a city realisation with $n=20$ and $\mathbf{u}=\left(m_{H}, m_{W}, m_{D}, \delta_{H}, \delta_{W}, \delta_{D}\right)=(10,20,30,0.2,0.2,0.2)$.

\section{Reference model: linear propagation over urban cities}

This section presents the stochastic linear propagation model used to obtain reference solutions of the problem. The problem setting (source and receiver, frequency range, ...) is first given in section 4.1 and the stochastic propagation model itself is then described in section 4.2.

\subsection{Setting the problem}

Output $P_{r}^{\exp }(\omega)$ from the reference model is analysed in the frequency domain on a frequency band defined by $B=] 0, \omega_{\max }$ ], in which $\omega_{\max }$ is such that $B$ is a low frequency band. Hence, the buildings surfaces are assumed to be acoustically rigid (no absorption and specular reflections). 
The source and receiver are placed above the urban city layer and the source is placed sufficiently far from the first building $B_{1}$ to assume that the wave impinging on this first building is a plane wave. The receiver is placed at the same altitude as the source and at the right of the last building $B_{n}$. The quantity under interest is the normalised spectrum $L^{\exp }(\omega)$ at the receiver such that

$$
L^{\exp }(\omega)=10 \log _{10}\left(\left|\frac{P_{r}^{\exp }(\omega)}{p_{\text {free }}(\omega)}\right|^{2}\right),
$$

where $p_{\text {free }}(\omega)$ and $P_{r}^{\exp }(\omega)$ denote free field pressure and the pressure in the presence of the urban city at the receiver, respectively. Note that the reference model used does not account for building surfaces vibrations.

\subsection{Stochastic BEM solver for constructing a reference solution}

The model used to construct the reference solution is an implementation of the boundary element method $[8,18](\mathrm{BEM})$, in which the city geometry is explicitly entered into the computational model. For a fixed parameter $\mathbf{u}$, the outputs $L^{\exp }(\omega)$ are calculated using the Monte Carlo method [16] with $v_{\text {exp }}$ independent realisations $\eta_{1}, \ldots, \eta_{v_{\text {exp }}}$ of urban cities generated with the probability models presented in section 3. The realisation of the experimental observation for the urban city $v_{p}$ is $\left\{L^{\exp }\left(\omega, \eta_{v_{p}}\right), \omega \in B\right\}$.

\section{Mean non-linear parabolic propagation model for sound propagation over urban cities}

This section describes the mean parabolic propagation model that will be later used, associated with a probabilistic model of uncertainties, to model sound propagation over urban environments. Section 5.1 presents a general overview of propagation models based on the non-linear parabolic equation (NPE), section 5.2 formally defines the propagation model and section 5.3 explains the choice of the propagation model parameters and details their algebraic properties.

\subsection{General overview of NPE models}

The non-linear parabolic equation (NPE) has first been developed by McDonald and Kuperman in 1987 [28] (see also [27, 6]) and has been successfully used for underwater acoustics simulations 
$[1,7]$ and blast wave propagation in air [40, 2, 21]. The NPE model for a 2D domain with Cartesian coordinates $(x, z)$ filed with air is:

$$
\partial_{t}^{\star} r(x, z ; t)=-\partial_{x}\left(c_{1} r(x, z ; t)+c_{0} \frac{\beta}{2} r(x, z ; t)^{2}\right)-\frac{c_{0}}{2} \int \partial_{z}^{2} r(x, z ; t) d x
$$

where $\partial_{i}$ means partial derivation with respect to variable $i, x$ is the main propagation direction, $z$ is the transverse propagation direction and $t$ is the time variable. The ambient sound speed is $c_{0}$ while $c_{1}$ is the sound speed perturbation in the moving window, i.e. $c_{1}=c(x, z)-c_{0}$, where $c(x, z)$ is the spatially-dependent sound speed. The dimensionless over-density variable is $r(x, z ; t)$ such that $r(x, z ; t)=\rho^{\prime}(x, z ; t) / \rho_{0}$, with $\rho^{\prime}(x, z ; t)$ the acoustic density perturbation and $\rho_{0}$ the ambient medium density. In the following the $(x, z ; t)$ dependence is dropped, i.e. $r(x, z ; t)$ is written $r$. For air, the coefficient of non-linearity $\beta$ is calculated with the help of the ratio of specific heats $\gamma$, i.e. $\beta=(\gamma+1) / 2$. The first term on the right hand side of equation (9) simulates refraction and non-linear effects. The second term accounts for propagation in the transverse direction. The moving window operator $\partial_{t}^{\star}$ is defined by

$$
\partial_{t}^{\star}=\partial_{t}+c_{0} \partial_{x}
$$

Note that in equation (9), the azimuthal spreading term $c_{0} r /(2 d)$, in which $d$ is the distance from the source to the point where the field is calculated, has been dropped from the original NPE [28]. There is no absorption from air included in the model. The NPE model derives from Euler's equations. The assumptions used are

(1) weak non-linearities, i.e. $\rho^{\prime} / \rho_{0} \ll 1$,

(2) weak sound speed perturbations, i.e. $c_{1} \ll c_{0}$,

(3) propagation along a main direction.

Various modifications and extensions to this original model were made during the past two decades. Spherical and cylindrical coordinate system versions [38] and high-angle formulation [26] have been developed. Too and Lee [39] has added a complementary term in the NPE equation in order to take into account thermoviscous effects. Propagation in multiple media [1] and propagation through atmospheric turbulences [4] were also successfully studied using this model. 
The NPE differential equation (9) is discretised with the finite difference method. The CrankNicolson method is used for linear terms yielding a tridiagonal system which can be solved with a Thomas algorithm (see for example [29]). For the non-linear term, one has to use a specialised algorithm which is able to propagate discontinuities and which is stable. In the numerical implementation used in this work, the flux corrected transport algorithm (FCT) [5, 42] is used. The principle of the FCT algorithm is to introduce some artificial viscosity in the numerical scheme to limit the Gibb oscillations.

In a previous work, the original NPE model has been extended to propagation within and over porous ground layers $[24,25]$. In this model the porous layer is assumed to be equivalent to a continuous fluid medium. A wave causes a vibration of air particles contained in the ground pores, while the ground frame does not vibrate. The model derivation and the applications to propagation over porous layers can be found in [23]. In this work, it is proposed to use this model to study long-range sound propagation over urban cities.

\subsection{NPE model for sound propagation in multiple media}

In this section, we summarise the NPE model for sound propagation in multiple media proposed in [23]. This propagation model is composed of three entities. The two first are non-linear parabolic equations for the air and the urban layers, and the third one is an interface condition to couple the two domains. Let $\Omega_{a}$ and $\Omega_{u}$ be two domains occupied by the atmosphere and the urban environment, and let $\Gamma_{a}$ be the boundary at the top of the domain and $\Gamma_{u}$ the boundary at the bottom. The coupling interface between the domains is noted $\Gamma$ and the boundary to the left and to the right of the domain $\Omega_{a} \cup \Omega_{u}$ are $\Gamma_{l}$ and $\Gamma_{r}$, respectively. The mean acoustic perturbation field at points $\mathbf{x}_{\mathbf{a}}=(x, z) \in \Omega_{a}$ and $\mathbf{x}_{\mathbf{u}}=(x, z) \in \Omega_{u}$ are $r_{a}$ and $r_{u}$, respectively. Figure 4 shows a sketch of the different domains with their boundaries. The NPE model for propagation in multiple media 


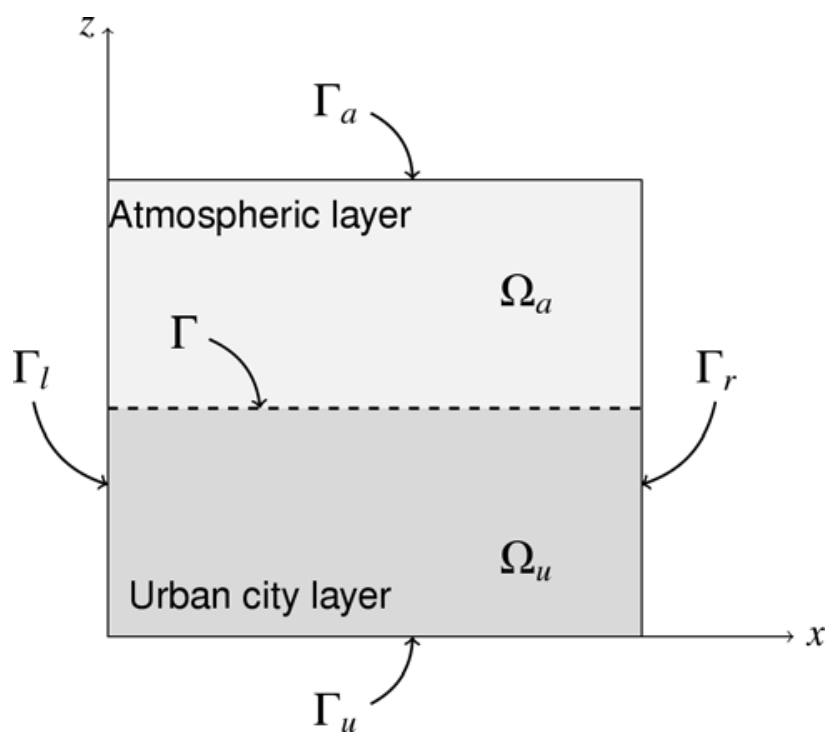

Figure 4: Sketch of the different domains with their respective boundaries of the NPE model for propagation in multiple media.

is written as

$$
\begin{array}{lr}
\partial_{t}^{\star} r_{a}+\partial_{x}\left(c_{1} r_{a}+\beta \frac{c_{0}}{2} r_{a}^{2}\right)+\frac{c_{0}}{2} \int \partial_{z}^{2} r_{a} d x=g\left(\mathbf{x}_{\mathbf{a}}, t\right) & \text { in } \Omega_{a}, \\
\partial_{t}^{\star} r_{u}+\partial_{x}\left(c_{0}(\mu-1) r_{u}+\beta \mu \frac{c_{0}}{2} r_{u}^{2}\right)+\frac{\mu c_{0}}{2} \int \partial_{z}^{2} r_{u} d x+\alpha r_{u}=0 & \text { in } \Omega_{u}, \\
\partial_{z} r_{a}+\epsilon \int \partial_{z} r_{a} d x=\gamma \partial_{z} r_{u} & \text { on } \Gamma, \\
r_{a}=0, r_{u}=0 & \text { on } \Gamma_{r}, \\
r_{a}, r_{u} \text { satisfy Sommerfeld radiation condition } & \text { on } \Gamma_{l}, \\
\partial_{z} r_{u}=0 & \text { on } \Gamma_{u}, \\
r_{a} \text { satisfies Sommerfeld radiation condition } & \text { on } \Gamma_{a},
\end{array}
$$

where $g\left(\mathbf{x}_{\mathbf{a}}, t\right)$ is an external pressure field applied in the atmospheric layer. Equations (11a) and (11b) handle propagation in the atmospheric and urban layers. Equation (11c) is the interface condition between the domains and equation (11d) is a standard boundary condition applied on the boundary $\Gamma_{r}$ to the right of the moving window. Equation (11e) states that the wave must not reflect at the left boundary of the domain, and hence the acoustic field must satisfy Sommerfeld radiation condition on $\Gamma_{l}$. It is supposed that the bottom of the domain is perfectly rigid, hence 
$\partial_{z} r_{u}=0$ on $\Gamma_{u}$ (see equation (11f)). Since the propagation problem is infinite in the $+z$ direction the acoustic field must satisfy Sommerfeld radiation condition on $\Gamma_{a}$ (equation $(11 \mathrm{~g})$ ). For the sake of brevity the explicit expressions of Sommerfeld radiation conditions are not given here. In the present work the propagation domain is truncated in the $+z$ direction with the help of a perfectly matched layer (see [3] and [9]).

Wave propagation in the urban layer (equation (11b)) is characterised by $\mu$ which modifies the sound speed in the urban layer so that $c_{\Omega_{u}}=\mu c_{\Omega_{a}}$, while $\alpha$ is the loss rate in the layer. The interface condition between domains $\Omega_{a}$ and $\Omega_{u}$ (equation (11c)) depends on parameters $\gamma$ and $\epsilon$. On the boundary $\Gamma_{r}$ a null pressure $r_{a}=0, r_{u}=0$ is imposed, meaning that no perturbation is introduced ahead of the wavefront. The relative sound pressure level at the receiver is given by

$$
L(\omega)=10 \log _{10}\left(\left|\frac{\rho_{0} c_{0}^{2} \widehat{r}_{a}\left(x_{r}, z_{r}, \omega\right)}{p_{\text {free }}\left(x_{r}, z_{r}, \omega\right)}\right|^{2}\right),
$$

where quantities with hats denote Fourier-transformed quantities, and $x_{r}$ and $z_{r}$ are the receiver coordinates.

\subsection{Construction of the mean model and description of the algebraic properties of its parameters}

Below the mean model is derived from section 5.2. One has to define the parameters in the mean propagation model (see equations (11)) which have the capability to represent the natural variability of the real system (the urban environment). These defined parameters will be modelled by random variables as explained in section 2 . When a wave is reflecting on a plane surface, its amplitude is changed and a (possibly negative) delay is given to the reflected wave. Parameters $(\mu, \alpha, \gamma, \epsilon)$ appearing in equations (11b) and (11c) could be used because the propagation model can recreate the behaviour of a porous ground layer [23] and could thus be used "as is" to control the wave reflection. However, in order to reduce the number of parameters in the mean model and consequently, to reduce the stochastic model complexity, another solution is proposed. The urban layer is now considered as semi-infinite, and one writes that no waves are transmitted from the porous ground layer to the atmospheric layer. This implies that the urban layer must behave like an atmospheric layer, which can be simulated in writing that $\mu=1$ and $\alpha=0$ in equation (11b). The time delay occurring during reflection is introduced by the use of a time-stretching. The time 
variable is then transformed according to

$$
t \rightarrow \frac{1}{\lambda} t
$$

and the time derivative is changed accordingly

$$
\partial_{t}^{\star} \longrightarrow \lambda \partial_{t}^{\star}
$$

The change of amplitude of the reflected wave is accounted for using the equation (11c). Parameter $\gamma$ can be used to tune the amount of reflected and transmitted waves. The time delay being already taken into account with the time stretching, there is no need to keep parameter $\epsilon$ in equation (11c). Parameter $\epsilon$ is set to $\epsilon=0$. One advantage of using such two parameters instead of four in the mean model, is that these two parameters $\gamma$ and $\lambda$ do not not introduce coupling effects between wave amplitude and time delay. This means that changing parameter $\lambda$ does not change the reflected wave amplitude, and changing parameter $\gamma$ does not change the time delay of the reflected wave. This property will later help designing the probabilistic model of these two parameters.

Note that parameter $\lambda$ cannot take the value 0 which would correspond to an infinite speed of sound. Parameter $\gamma$ is used to tune the amount of reflected wave in the atmospheric layer. Setting $\gamma=0$ yields $\partial_{z} r_{a}=0$ on coupling interface $\Gamma$, which is the condition for perfect reflection. Setting $\gamma=1$ yields $\partial_{z} r_{a}=\partial_{z} r_{u}$ on the coupling interface $\Gamma$, which is the condition for perfect transmission; parameter $\gamma$ thus belongs to $[0,1]$. Introducing underlined quantities related to the mean model, the NPE model for propagation in multiple media with the two above parameters is rewritten as

$$
\begin{array}{lc}
\underline{\lambda} \partial_{t}^{\star} \underline{r_{a}}+\partial_{x}\left(c_{1} \underline{r_{a}}+\beta \frac{c_{0}}{2} \underline{r_{a}}\right)+\frac{c_{0}}{2} \int \partial_{z}^{2} \underline{r_{a}} d x=g\left(\mathbf{x}_{\mathbf{a}}, t\right) & \text { in } \Omega_{a}, \\
\underline{\lambda} \partial_{t}^{\star} \underline{r_{u}}+\partial_{x}\left(\beta \frac{c_{0}}{2} \underline{r}_{u}^{2}\right)+\frac{c_{0}}{2} \int \partial_{z}^{2} \underline{r_{u}} d x=0 & \text { in } \Omega_{u}, \\
\partial_{z} \underline{r_{a}}=\underline{\gamma} \partial_{z} \underline{r_{u}} & \text { on } \partial \Omega, \\
\underline{r_{a}}=0, \underline{r_{u}}=0 & \text { on } \Gamma_{r}, \\
\underline{r_{a}} \text { and } \underline{r_{u}} \text { satisfy Sommerfeld radiation condition } & \text { on } \Gamma_{l}, \\
\underline{r_{u}} \text { satisfies Sommerfeld radiation condition } & \text { on } \Gamma_{u}, \\
\underline{r_{a}} \text { satisfies Sommerfeld radiation condition } & \text { on } \Gamma_{a} .
\end{array}
$$


Furthermore, introducing a third parameter $\underline{\theta}$ in order to control the output of the mean model, the relative sound pressure level at the receiver is given by

$$
\underline{L}(\omega)=10 \log _{10}\left(\left|\frac{\rho_{0} c_{0}^{2} \underline{\hat{r}_{a}}\left(x_{r}, z_{r}, \omega+\underline{\theta}\right)}{p_{\text {free }}\left(x_{r}, z_{r}, \omega+\underline{\theta}\right)}\right|^{2}\right),
$$

in which $\underline{\theta}$ is a real and positive frequency shifting parameter. Its only intent is to help controlling the mean model output. The mean propagation model parameter $\underline{\mathbf{s}}$ can now be defined as

$$
\underline{\mathbf{s}}=(\underline{\gamma}, \underline{\lambda}, \underline{\theta})
$$

Vector $\underline{\mathbf{s}}$ belongs to the admissible set $\mathcal{S}=([0,1] \times] 0,+\infty[\times[0,+\infty[)$. The initial value of $\underline{\mathbf{s}}$ is arbitrarily chosen as $\underline{\mathbf{s}}^{\mathbf{0}}=\left(\underline{\gamma}^{0}, \underline{\lambda}^{0}, \underline{\theta}^{0}\right)=(0,1,0)$ in which the superscript 0 refers to the nominal value of $\underline{\mathbf{s}}$, so that the nominal mean model simulates a wave reflecting on a plane which is an acoustically rigid surface.

\section{Construction of the probabilistic model of random variables $\Gamma, \Lambda$ and $\Theta$}

Let $\Gamma, \Lambda$ and $\Theta$ be the random variables associated with the mean model parameters $\underline{\gamma}, \underline{\lambda}$ and $\underline{\theta}$. Information Theory [33] and the Maximum Entropy Principle[17] are used to construct their probability distributions (see section 3).

\subsection{Construction of the probability distribution of random variable $\Gamma$}

The available information for random variable $\Gamma$ is the following.

(1) $\Gamma$ is a random variable with values in $[0,1]$ (see section 5.3).

(2) Its mean value $m_{\Gamma}=E\{\Gamma\}$ is given.

(3) Its coefficient of variation $\delta_{\Gamma}$ is given.

It should be noted that the upper bound $\gamma=1$ corresponds to perfect reflection at the interface and the lower bound $\gamma=0$ corresponds to perfect transmission. Since the neighbourhoods of these two bounds can be reached with a non-zero probability, it is not necessary to introduce an available information related to the behaviour of the probability distribution in the neighbourhood of these two bounds. With such an available information, the Maximum Entropy Principle yields

$$
p_{\Gamma}(\gamma)=\mathbb{1}_{[0,1]}(\gamma) e^{-\mu_{0}-\gamma \mu_{1}-\gamma^{2} \mu_{2}},
$$


where the constants $\mu_{0}, \mu_{1}$ and $\mu_{2}$ depend on $m_{\Gamma}$ and $\delta_{\Gamma}$ and are the solutions of the equations

$$
\begin{aligned}
& \int_{0}^{1} \gamma e^{-\gamma \mu_{1}-\gamma^{2} \mu_{2}} d \gamma-m_{\Gamma} \int_{0}^{1} e^{-\gamma \mu_{1}-\gamma^{2} \mu_{2}} d \gamma=0, \\
& \int_{0}^{1} \gamma^{2} e^{-\gamma \mu_{1}-\gamma^{2} \mu_{2}} d \gamma-\left(m_{\Gamma}^{2}+\sigma_{\Gamma}^{2}\right) \int_{0}^{1} e^{-\gamma \mu_{1}-\gamma^{2} \mu_{2}} d \gamma=0, \\
& \int_{0}^{1} e^{-\gamma \mu_{1}-\gamma^{2} \mu_{2}} d \gamma-e^{\mu_{0}}=0 .
\end{aligned}
$$

In the equations above, integrals are numerically evaluated with the Monte Carlo method and the equations are solved using a non-linear Least-Squares method [13].

\subsection{Construction of the probability distribution of random variable $\Lambda$}

Taking into account the algebraic properties given in section 5.3, random variable $\Lambda$ is with values in $] 0,+\infty$ [. Since $\lambda$ cannot take the value 0 which would correspond to an infinite speed of sound, it is necessary to write that the probability distribution goes sufficiently fast to zero when $\lambda$ goes to zero with superior values. Such a property is satisfied in introducing the following condition,

$$
E\{\log \Lambda\}=c \quad, \quad|c|<+\infty \quad
$$

which implies that the inverse $\Lambda^{-1}$ of $\Lambda$ is a second-order random variable. Therefore, the available information for random variable $\Lambda$ is the following.

(1) $\Lambda$ is a random variable with values in $] 0,+\infty[$.

(2) Its mean value $m_{\Lambda}=E\{\Lambda\}$ is given.

(3) Equation (20) is satisfied.

With the constraints defined by the above available information, the use of the Maximum Entropy Principle yields (see section 3),

$$
p_{\Lambda}(\lambda)=\mathbb{1}_{] 0,+\infty[}(\lambda) \frac{1}{m_{\Lambda}}\left(\frac{1}{\delta_{\Lambda}^{2}}\right) \frac{1}{\Gamma\left(1 / \delta_{\Lambda}^{2}\right)}\left(\frac{\lambda}{m_{\Lambda}}\right)^{\frac{1}{\delta_{\Lambda}^{2}}-1} \exp \left(-\frac{\lambda}{\delta_{\Lambda}^{2} m_{\Lambda}}\right),
$$

in which $\delta_{\Lambda}$ is the coefficient of variation of $\Lambda$. 


\subsection{Construction of the probability distribution of random variable $\Theta$}

The available information relative to random variable $\Theta$ is the same as the available information defined in section 6.2 for random variable $\Lambda$. Consequently, the probability density function of random variable $\Theta$ is written as

$$
p_{\Theta}(\theta)=\mathbb{1}_{] 0,+\infty[}(\theta) \frac{1}{m_{\Theta}}\left(\frac{1}{\delta_{\Theta}^{2}}\right) \frac{1}{\Gamma\left(1 / \delta_{\Theta}^{2}\right)}\left(\frac{\theta}{m_{\Theta}}\right)^{\frac{1}{\delta_{\Theta}^{2}}-1} \exp \left(-\frac{\theta}{\delta_{\Theta}^{2} m_{\Theta}}\right),
$$

in which $m_{\Theta}$ and $\delta_{\Theta}$ are the mean value and the coefficient of variation of random variable $\Theta$.

\section{Stochastic non-linear propagation over urban cities}

The following vector $\mathbf{w}$ of the parameters of the probabilistic models of random variables $\Gamma, \Lambda$ and $\Theta$ is introduced

$$
\mathbf{w}=\left(m_{\Gamma}, m_{\Lambda}, m_{\Theta}, \sigma_{\Gamma}, \sigma_{\Lambda}, \sigma_{\Theta}\right)
$$

in which $\sigma_{\Gamma}=m_{\Gamma} \delta_{\Gamma}, \sigma_{\Lambda}=m_{\Lambda} \delta_{\Lambda}$ and $\sigma_{\Theta}=m_{\Theta} \delta_{\Theta}$. Parameter $\mathbf{w}$ belongs to the admissible set $\mathcal{W}=(] 0,+\infty[)^{6}$. The stochastic model for non-linear sound propagation over urban cities is defined by

$$
\begin{array}{lr}
\Lambda \partial_{t}^{\star} R_{a}+\partial_{x}\left(c_{1} R_{a}+\beta \frac{c_{0}}{2} R_{a}^{2}\right)+\frac{c_{0}}{2} \int \partial_{z}^{2} R_{a} d x=g & \text { in } \Omega_{a}, \\
\Lambda \partial_{t}^{\star} R_{u}+\partial_{x}\left(\beta \frac{c_{0}}{2} R_{u}^{2}\right)+\frac{c_{0}}{2} \int \partial_{z}^{2} R_{u} d x=0 & \text { in } \Omega_{u}, \\
\partial_{z} R_{a}=\Gamma \partial_{z} R_{u} & \text { on } \Gamma, \\
R_{a}=0, R_{u}=0 & \text { on } \Gamma_{r}, \\
R_{a} \text { and } R_{u} \text { satisfy Sommerfeld radiation condition } & \text { on } \Gamma_{l}, \\
R_{u} \text { satisfies Sommerfeld radiation condition } & \text { on } \Gamma_{u}, \\
R_{a} \text { satisfies Sommerfeld radiation condition } & \text { on } \Gamma_{a} .
\end{array}
$$

The relative sound pressure level is calculated with

$$
L(\omega, \mathbf{w})=10 \log _{10}\left(\left|\frac{\rho_{0} c_{0}^{2} \widehat{R}_{a}\left(x_{r}, z_{r}, \omega+\Theta\right)}{p_{\text {free }}\left(x_{r}, z_{r}, \omega+\Theta\right)}\right|^{2}\right) .
$$


In equations (24) and (25), the probability distributions of random variables $\Gamma, \Lambda$ and $\Theta$ depend on $\mathbf{w}$, which in turn depends on $\mathbf{u}$, the parameter that describes the urban city geometry. Hence, to complete the construction of the computational model and to obtain observations of $L(\omega, \mathbf{w})$, one has to express parameter $\mathbf{w}$ as a function of parameter $\mathbf{u}$ (it should be noted that no explicit expression can be constructed but the corresponding mapping will be numerically constructed).

\section{Identification of parameter $w$ of the stochastic model}

\subsection{Identification strategy}

The identification of parameter $\mathbf{w}$ is performed by solving an inverse stochastic problem. Mean-square methods [36, 43] and the maximum likelihood method [35] are generally used to solve such a problem. Both techniques have been tested (see below) for different values of parameter $\mathbf{u}$ (corresponding to different urban environments). The conclusions of this comparative study are the following.

(i) Since the mean-square methods introduce a mean-square distance between the experimental data and the random response of the stochastic model, this type of method is equivalent to a minimisation of the sum of the variance for the stochastic model response with the bias between the experimental mean value and the mean value of the random response. If these two mean values are significantly different, the bias can only be reduced in increasing the variance of the model. In this case, the distance between the experimental mean value and the mean value of the random response of the stochastic model is effectively reduced but in counterpart, the confidence region of the random response increases (see for example [12]).

(ii) For the application analysed it has been seen that, for certain values of parameter $\mathbf{u}$, the maximum likelihood method under-estimates the width of the confidence region which means that an important number of experimental paths cross the upper and the lower envelopes of the confidence region. This indicates that the maximum likelihood method cannot lead to an accurate description of the sound field for certain values of parameter $\mathbf{u}$.

To provide an accurate and robust identification method for all values of parameter $\mathbf{u}$, a hybrid method is used; the identification of the optimal parameter $\mathbf{w}^{\text {opt }}$ is done in two successive steps: 
(1) The mean model is first updated with experimental data: this step consists of finding the optimal mean model parameter $\underline{\mathbf{s}}^{\text {opt }}$ that minimises the norm between the mean value of the reference model outputs and the mean model output. As only the mean value of the experimental observations is used, this first step only allows us to identify the parameter that optimises the mean response of the stochastic model.

(2) Next, to take into account the dispersion of the experimental observations, a multi-objective optimisation problem is solved: both the mean-square and the maximum likelihood methods are used concurrently. This multi-objective optimisation problem aims to find the optimal parameter $\mathbf{w}^{\text {opt }}$ that maximises the log-likelihood function between the experimental observations and the stochastic model outputs, while minimising the areas where experimental observations do not belong to the confidence regions of the stochastic model. This problem is solved using an evolutionary algorithm $[10,37]$.

Step (1) allows us to obtain a rough approximation of the mean values $m_{\Gamma}, m_{\Lambda}$ and $m_{\Theta}$ which are the three first components of vector $\mathbf{w}$, and hence helps initialising the optimisation problem in step (2). Section 8.2 presents the procedure to update the mean model with experimental data (step (1)). The mean square method and the maximum likelihood method are presented in sections 8.3 and 8.4 .

\subsection{Updating the mean model with experimental data}

The observation from the mean model depending on the choice of parameter $\underline{\mathbf{s}}$, it is rewritten as $\underline{L} \underline{\underline{\mathrm{s}}}(\omega)$. The performance level of the nominal model can be measured in estimating the norm

$$
\left\|E\left\{L^{\exp }\right\}-\underline{L}^{\mathrm{s}}\right\|_{B}=\left\{\int_{\omega \in B}\left|E\left\{L^{\exp }(\omega)\right\}-\underline{L}^{\underline{\mathrm{s}}}(\omega)\right|^{2} d \omega\right\}^{1 / 2},
$$

and the nominal value of the mean model parameter $\underline{\mathbf{s}}^{\mathbf{0}}$ can be updated in a vector $\underline{\mathbf{s}}^{\text {opt }}$ such that

$$
\underline{\mathbf{s}}^{\mathrm{opt}}=\arg \min _{\underline{\mathbf{s}} \in \mathcal{S}}\left\|E\left\{L^{\mathrm{exp}}\right\}-\underline{L} \underline{\mathrm{s}}^{\mathrm{p}}\right\|_{B}
$$

Hence for a fixed value of $\mathbf{u}=\left(m_{H}, m_{W}, m_{D}, \delta_{H}, \delta_{W}, \delta_{D}\right)$, the parameter $\underline{\mathbf{s}}^{\text {opt }}$ that minimises the norm between the mathematical expectation of the experimental observations and the observation from the mean model can be determined. 


\subsection{Identification of the stochastic model parameter with the mean-square method}

This section presents the mean-square method [36, 43] with non-differentiable objective function [35] for the identification of the parameter $\mathbf{w}^{\mathrm{opt}}$ of the stochastic model. The objective function is defined by writing that the $\eta_{v_{\exp }}$ experimental observations $\left\{L^{\exp }\left(\omega ; \eta_{j}\right), \omega \in B, j=1, \ldots, v_{\exp }\right\}$, must belong to the confidence region of the stochastic model with a probability level $P_{c}$ fixed in ] 0,1 [. Before giving the formal definition of the mean-square method one must address the problem of the construction of confidence region.

The confidence region is constructed by using the quantiles. Let $F_{L(\omega)}$ be the cumulative distribution function of random variable $L(\omega)$, such that

$$
F_{L(\omega)}(\ell)=\mathrm{P}\{L(\omega) \leq \ell\} \quad .
$$

In equation (28) the right-hand side represents the probability that the random variable $L(\omega)$ takes on a value less than or equal to $\ell$. For $0<p<1$, the $\mathrm{p}^{\text {th }}$ quantile (or fractile) of $F_{L(\omega)}$ is defined as

$$
\zeta(p ; \omega)=\inf _{\ell}\left\{F_{L(\omega)}(\ell) \geq p\right\}
$$

Then, the upper and lower envelopes $\ell^{+}(\omega)$ and $\ell^{-}(\omega)$ of the confidence region are given by

$$
\ell^{+}(\omega)=\zeta\left(\frac{1+P_{c}}{2} ; \omega\right) \quad, \quad \ell^{-}(\omega)=\zeta\left(\frac{1-P_{c}}{2} ; \omega\right) .
$$

The estimation of $\ell^{+}(\omega)$ and $\ell^{-}(\omega)$ is performed by using the sample quantile [32]. Consider $v$ independent realisations of the random variable $L(\omega)$ noted $\ell_{1}(\omega)=L\left(\omega ; \eta_{1}\right), \ldots, \ell_{v}(\omega)=$ $L\left(\omega ; \eta_{v}\right)$ and let $\widetilde{\ell}_{1}(\omega)<\ldots<\widetilde{\ell}_{v}(\omega)$ be the ordered statistics associated with $\ell_{1}(\omega), \ldots, \ell_{v}(\omega)$. One has the following estimations for the upper and lower envelopes:

$$
\begin{array}{ll}
\ell^{+}(\omega) \simeq \widetilde{\ell}_{j^{+}}^{+}(\omega), & j^{+}=\operatorname{fix}\left(v \frac{1+P c}{2}\right), \\
\ell^{-}(\omega) \simeq \widetilde{\ell_{j^{-}}^{-}}(\omega), & j^{-}=\operatorname{fix}\left(v \frac{1-P c}{2}\right),
\end{array}
$$

in which fix $(x)$ is the integer part of the real number $x$.

Now introducing the dependence on parameter $\mathbf{w}$, the formal definition of the mean-square method now follows. Let $\ell^{+}(\mathbf{w}, \omega)$ and $\ell^{-}(\mathbf{w}, \omega)$ be the upper and lower envelopes of the confidence region of the stochastic model. The functions $\ell^{+\exp }(\omega), \ell^{-\exp }(\omega), z^{+}(\mathbf{w}, \omega)$ and $z^{-}(\mathbf{w}, \omega)$ 
are such that

$$
\begin{gathered}
\ell^{+\exp }(\omega)=\max _{j} L^{\exp }\left(\omega ; \eta_{j}\right) \quad, \quad \ell^{-\exp }(\omega)=\min _{j} L^{\exp }\left(\omega ; \eta_{j}\right), \\
z^{+}(\mathbf{w}, \omega)=\left(\ell^{+}(\mathbf{w}, \omega)-\ell^{+\exp }(\omega)\right) \times\left(1-\mathrm{H}\left(\ell^{+}(\mathbf{w}, \omega)-\ell^{+\exp }(\omega)\right)\right), \\
z^{-}(\mathbf{w}, \omega)=\left(\ell^{-}(\mathbf{w}, \omega)-\ell^{-\exp }(\omega)\right) \times\left(1-\mathrm{H}\left(\ell^{-}(\mathbf{w}, \omega)-\ell^{-\exp }(\omega)\right)\right),
\end{gathered}
$$

in which $\mathrm{H}(x)$ is the Heaviside function such that $\mathrm{H}(x)=1$ if $x \geq 0$ and $\mathrm{H}(x)=0$ otherwise. Functions $z^{+}(\mathbf{w}, \omega)$ and $z^{-}(\mathbf{w}, \omega)$ represent selected parts of $\left[\ell^{+}(\mathbf{w}, \omega)-\ell^{+\exp }(\omega)\right]$ and $\left[\ell^{-}(\mathbf{w}, \omega)-\ell^{-\exp }(\omega)\right]$ where the experimental observations do not belong to the confidence region calculated with the stochastic model. The non-differentiable objective function $J(\mathbf{w})$ is then defined by

$$
J(\mathbf{w})=\left\|z^{+}(\mathbf{w}, .)\right\|_{B}^{2}+\left\|z^{-}(\mathbf{w}, .)\right\|_{B}^{2}
$$

and the optimal parameter $\mathbf{w}^{\text {opt }}$ is solution of the following optimisation problem:

$$
\mathbf{w}^{\mathrm{opt}}=\arg \min _{\mathbf{w} \in \mathcal{W}} J(\mathbf{w})
$$

The mean-square method aims to minimise the areas where the experimental observations do not belong to the confidence region of the stochastic model: the only criteria to select the optimal parameter $\mathbf{w}^{\mathrm{opt}}$ is hence the amount of information not covered by the stochastic model.

\subsection{Identification of the stochastic model parameter with the maximum likelihood method and} statistical reduction of information

This section deals with the maximum likelihood method to identify the optimal parameter $\mathbf{w}^{\text {opt }}$. Let $\left\{\omega_{1}, \ldots, \omega_{m}\right\} \subset B$ be a sampling of frequency band $B$ and let $\mathbf{w} \rightarrow \mathcal{L}(\mathbf{w})$ be the log-likelihood function from $\mathcal{W}$ into $\mathbb{R}$, defined by

$$
\mathcal{L}(\mathbf{w})=\sum_{j=1}^{v_{\text {exp }}} \log _{10} p\left(L^{\exp }\left(\omega_{1} ; \eta_{j}\right), \ldots, L^{\exp }\left(\omega_{m} ; \eta_{j}\right) ; \mathbf{w}\right),
$$

in which $p\left(L^{\exp }\left(\omega_{1} ; \eta_{j}\right), \ldots, L^{\exp }\left(\omega_{m} ; \eta_{j}\right) ; \mathbf{w}\right)$ is the joint probability density function of random variables $L\left(\omega_{1}, \mathbf{w}\right), \ldots, L\left(\omega_{m}, \mathbf{w}\right)$ for the values $L^{\exp }\left(\omega_{1} ; \eta_{j}\right), \ldots, L^{\exp }\left(\omega_{m} ; \eta_{j}\right)$. The maximum 
likelihood method [36] consists in finding $\mathbf{w}^{\text {opt }}$ as the solution of the following optimisation problem:

$$
\mathbf{w}^{\mathrm{opt}}=\arg \max _{\mathbf{w} \in \mathcal{W}} \mathcal{L}(\mathbf{w})
$$

The standard method requires a direct evaluation of the joint probability density function appearing in equation (36) which involves a significant computational effort. In order to decrease this effort the method of statistical reduction of information introduced in [35] is used. The principle of the method is to proceed to a statistical reduction of information using a principal component analysis (see for instance [19]) and then to use the maximum likelihood method in the space of the uncorrelated random variables related to the reduced statistical information.

For all $\mathbf{w}$ fixed in $\mathcal{W}$, let be $\mathbf{L}(\mathbf{w})=\left(L\left(\omega_{1}, \mathbf{w}\right), \ldots, L\left(\omega_{m}, \mathbf{w}\right)\right)$. Let $\mathbf{m}(\mathbf{w})=E\{\mathbf{L}(\mathbf{w})\}$ be its mean value and let $\left[C_{\mathbf{L}(\mathbf{w})}\right]$ be its $(m \times m)$ covariance matrix defined by

$$
\left[C_{\mathbf{L}(\mathbf{w})}\right]=E\left\{(\mathbf{L}(\mathbf{w})-\mathbf{m}(\mathbf{w}))(\mathbf{L}(\mathbf{w})-\mathbf{m}(\mathbf{w}))^{T}\right\},
$$

in which $T$ superscript stands for transposition. The following eigenvalue problem is introduced

$$
\left[C_{\mathbf{L}(\mathbf{w})}\right] \mathbf{x}(\mathbf{w})=e(\mathbf{w}) \mathbf{x}(\mathbf{w}),
$$

for which the first largest $q \leq m$ positive eigenvalues are $e_{1}(\mathbf{w}) \geq e_{2}(\mathbf{w}) \geq \ldots \geq e_{q}(\mathbf{w})$ and the associated eigenvectors are $\mathbf{x}^{1}(\mathbf{w}), \ldots, \mathbf{x}^{q}(\mathbf{w})$, in $\mathbb{R}^{m}$. The approximation $\mathbf{L}^{q}(\mathbf{w})$ of $\mathbf{L}(\mathbf{w})$ is written as

$$
\mathbf{L}^{q}(\mathbf{w})=\mathbf{m}(\mathbf{w})+\sum_{\alpha=1}^{q} \sqrt{e_{\alpha}(\mathbf{w})} Y_{\alpha}(\mathbf{w}) \mathbf{x}^{\alpha}(\mathbf{w}),
$$

in which $Y_{1}(\mathbf{w}), \ldots, Y_{q}(\mathbf{w})$ are $q$ real-valued random variables such that, for all $\alpha=1, \ldots, q$

$$
Y_{\alpha}(\mathbf{w})=\frac{1}{\sqrt{e_{\alpha}(\mathbf{w})}} \mathbf{x}^{\alpha}(\mathbf{w})^{T}(\mathbf{L}(\mathbf{w})-\mathbf{m}(\mathbf{w}))
$$

It can easily be proved that $\mathbf{Y}(\mathbf{w})=\left(Y_{1}(\mathbf{w}), \ldots, Y_{q}(\mathbf{w})\right)$ is a second-order random variable such that, for all $\alpha$ and $\beta$ in $\{1 \ldots q\}$

$$
E\left\{Y_{\alpha}(\mathbf{w})\right\}=0 \quad, \quad E\left\{Y_{\alpha}(\mathbf{w}) Y_{\beta}(\mathbf{w})\right\}=\delta_{\alpha \beta},
$$


which means that the centred random variables $Y_{1}(\mathbf{w}), \ldots, Y_{q}(\mathbf{w})$ are uncorrelated. The order $q$ of the statistical reduction is calculated in order to get an approximation with a given accuracy $\epsilon$ which has to be chosen such that

$$
\max _{\mathbf{w} \in \mathcal{W}}\left\{1-\frac{\sum_{\alpha=1}^{q} e_{\alpha}(\mathbf{w})}{\operatorname{tr}\left[C_{\mathbf{L}}(\mathbf{w})\right]}\right\} \leq \epsilon
$$

From equation (41) one can deduce that random variables $Y_{\alpha}^{\exp }\left(\mathbf{w} ; \eta_{j}\right)$ associated with the experimental realisation $\eta_{j}$ are given, for all $\alpha=1, \ldots, q$ and $j=1, \ldots, v_{\text {exp }}$ by

$$
Y_{\alpha}^{\exp }\left(\mathbf{w} ; \eta_{j}\right)=\frac{1}{\sqrt{e_{\alpha}(\mathbf{w})}} x^{\alpha}(\mathbf{w})^{T}\left(\mathbf{L}^{\exp }\left(\eta_{j}\right)-\mathbf{m}(\mathbf{w})\right)
$$

Finally, taking into account that the random variables $Y_{1}(\mathbf{w}), \ldots, Y_{q}(\mathbf{w})$ are mutually independent, one introduces the following approximation for the reduced $\log$-likelihood function $\mathcal{L}^{\text {red }}$,

$$
\mathcal{L}^{\mathrm{red}}(\mathbf{w})=\sum_{j=1}^{v_{\text {exp }}} \sum_{\alpha=1}^{q} \log _{10} p_{Y_{\alpha}(\mathbf{w})}\left(Y_{\alpha}^{\exp }\left(\mathbf{w} ; \eta_{j}\right) ; \mathbf{w}\right)
$$

where $p_{Y_{\alpha}(\mathbf{w})}(y ; \mathbf{w})$ is the probability density function of the real-valued random variable $Y_{\alpha}(\mathbf{w})$. The optimal value $\mathbf{w}^{\text {opt }}$ of $\mathbf{w}$ is then given by,

$$
\mathbf{w}^{\mathrm{opt}}=\arg \max _{\mathbf{w} \in \mathcal{W}} \mathcal{L}^{\mathrm{red}}(\mathbf{w})
$$

This problem is solved by using a genetic algorithm based on non-dominated sorting (NSGAII) $[10,37]$. This method is used to solve the multi-objective optimisation problem for which an initial population evolves over several generations of individuals. For each generation a selection process selects the "best" parents from which children are generated.

\section{Application and numerical validation}

It is proposed in this section to use the here developed stochastic parabolic propagation model to study sound propagation over given urban environments. Numerical solutions from a BEM code are used as references. 


\subsection{Summary of previous sections - Stochastic model validation procedure}

In order to construct and validate the stochastic propagation model one has to

(1) choose a parameter $\mathbf{u}=\left(m_{H}, m_{W}, m_{D}, \delta_{H}, \delta_{W}, \delta_{D}\right)$ that describes the urban city geometry studied. With this parameter the $v_{\exp }$ urban environment realisations can be generated with the probability distributions given in section 3 (see section 9.2).

(2) using the $v_{\text {exp }}$ realisations of the probabilistic model of the given city, and using the stochastic reference model (see section 4), produce the family $\left\{L^{\exp }\left(\omega ; \eta_{j}\right), \omega \in B\right\}_{j}$ (see section 9.2).

(3) once the experimental realisations are obtained, identify the optimal parameter $\mathbf{w}^{\text {opt }}$ of the stochastic model with the help of the method described in section 8 (section 9.3).

(4) using the stochastic parabolic propagation model derived from section 7 , construct the confidence region associated with the optimal parameter $\mathbf{w}^{\text {opt }}$ determined in (3) which finally allows the stochastic propagation model to be validated (section 9.4).

\subsection{Choice of parameter $\mathbf{u}$, numerical experiment description and output calculation from the reference model}

As explained in section 4, the reference model is defined as a numerical experiment consisting of numerical calculations using the Boundary Element Method (BEM). The source and receiver are positioned $2000 \mathrm{~m}$ from the central point of the urban environment at an angle of $5^{\circ}$ and the urban environment is composed of $n=20$ buildings. The ground and building surfaces are supposed acoustically rigid and computations are performed on a frequency range $B=] 0,100 \mathrm{~Hz}$ with a frequency sampling $\Delta f=2 \mathrm{~Hz}$ yielding data vectors with 51 values. Figure 5 shows a sketch of the configuration.

In this application two different city morphologies are chosen. The corresponding parameters are $\mathbf{u}_{\mathbf{1}}$ and $\mathbf{u}_{\mathbf{2}}$ such that $\mathbf{u}_{\mathbf{1}}=\left(m_{H}, m_{W}, m_{D}, \delta_{H}, \delta_{W}, \delta_{D}\right)=(10,20,30,0.2,0.2,0.2)$ and $\mathbf{u}_{2}=$ $(40,40,30,0.2,0.2,0.2)$. Once $\mathbf{u}_{1}$ and $\mathbf{u}_{2}$ are fixed the buildings dimensions are generated with the help of the probability distributions defined in section 3 . The number of realisations of the probabilistic model of the real urban city is $v_{\exp }=500$, inducing a convergence with a $10^{-3}$ accuracy (at least) for the first and second order moment of the random variable $\mathbf{L}^{\exp }$. A calculation with 


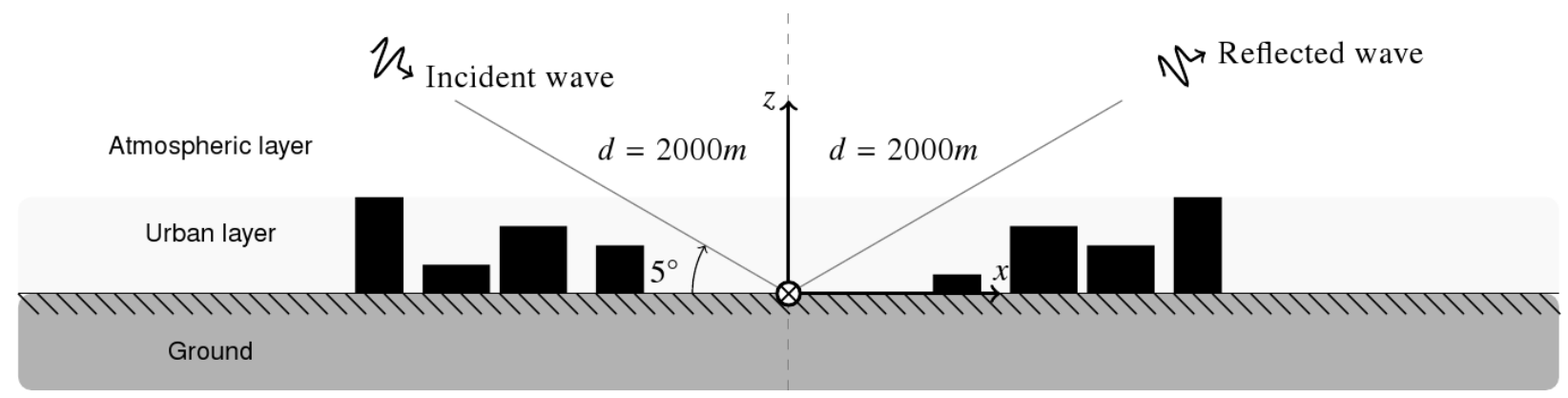

Figure 5: Sketch for the reference model (numerical experiment).

the nominal mean model described in section 5 is also performed for comparison and is initialised with

$$
\underline{\mathbf{s}}^{\mathbf{0}}=\left(\underline{\gamma}^{0}, \underline{\lambda}^{0}, \underline{\theta}^{0}\right)=(0,1,0),
$$

for which the interface behaves like a plane acoustically rigid surface. Figure 6 compares the reference model (made up of 500 outputs and the mean values estimates $m_{L_{1}^{\exp }}$ and $m_{L_{2}^{\exp }}$ ) with the nominal model output $\underline{L}^{\mathbf{s}^{0}}$.

For the value $\mathbf{u}_{\mathbf{1}}$ of the parameter describing the urban environment, a low dispersion on the observations and quasi-specular reflection behaviour are obtained. The output from the nominal model and the mean value of the reference model output have a similar shape. For the value $\mathbf{u}_{2}$ of the parameter describing the urban environment, low dispersion on the low frequency range and high dispersion in the range $[65,90] \mathrm{Hz}$ can be observed. It can also be noted that the mean value estimate of the experimental realisations $L_{2}^{\exp }(\omega)$ is contained in a relatively narrow region. The maximum and minimum values never exceed $6 \mathrm{~dB}$ and $-6 \mathrm{~dB}$. The shape of the experimental realisations differs from the shape of the nominal model. As it can be seen in figure 6 the output from the mean model $\underline{L}^{\mathbf{s}^{0}}$ has misplaced interference dips for both urban environments parameters $\mathbf{u}_{1}$ and $\mathbf{u}_{2}$.

\subsection{Identification of the stochastic model optimal parameter $\mathbf{w}^{\text {opt }}$}

In this section, the procedure described in section 8 is used to identify the optimal parameters $\mathbf{w}_{1}^{\text {opt }}$ and $\mathbf{w}_{2}^{\text {opt }}$ of the stochastic model corresponding to the parameters $\mathbf{u}_{\mathbf{1}}$ and $\mathbf{u}_{\mathbf{2}}$. 


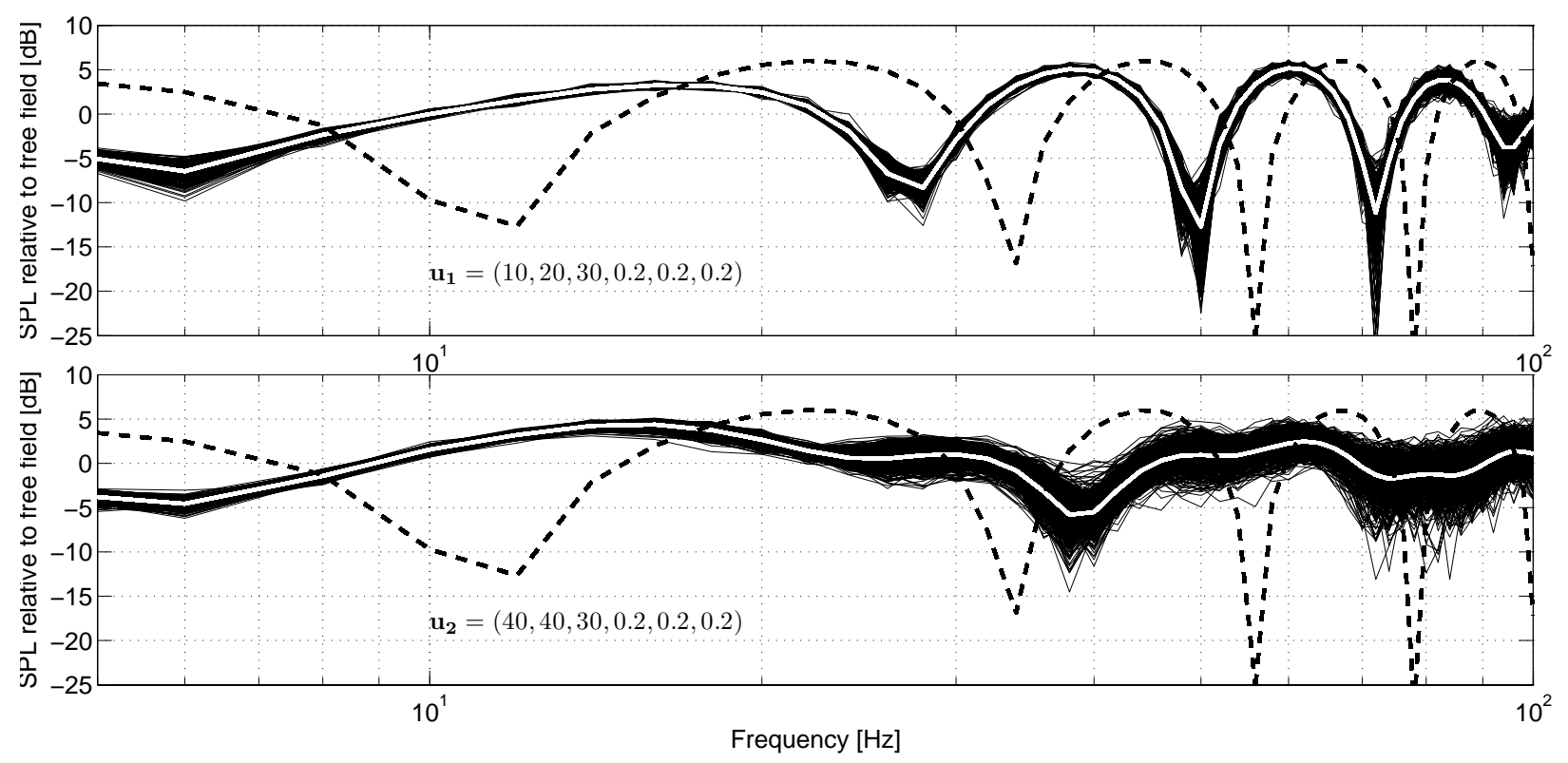

Figure 6: Comparisons of the reference model with the nominal model (initial mean model). Reference model (made up of 500 outputs (thin lines) and the mean values estimates $m_{L_{1}^{\exp }}$ and $m_{L_{2}^{\exp }}$ (thick white lines)). Nominal model output $\underline{L}^{\mathbf{0}}$ (dashed line). The value of parameter $\mathbf{u}$ is $\mathbf{u}_{1}$ (top figure) and $\mathbf{u}_{2}$ (bottom figure).

\subsubsection{Determination of the updated parameter of the mean model}

In the identification of the optimal parameter $\mathbf{w}^{\text {opt }}$ of the stochastic model, the first step is the determination of the updated parameter $\underline{\mathbf{s}}^{\text {opt }}$ of the mean model (step 1 in section 8.2), solution of the minimisation problem defined in equation 27.

The effects of parameters $\underline{\gamma}, \underline{\lambda}$ and $\underline{\theta}$ on the reflected wave being uncoupled, the minimisation problem is reduced to three one-dimensional searches. The parameters are real and we use a parabolic interpolation and the Brent's method [30]. The optimal parameter found from this minimisation problem is $\underline{\mathbf{s}}_{1}^{\mathrm{opt}}=\left(\underline{\gamma}_{1}^{\mathrm{opt}}, \underline{\lambda}_{1}^{\mathrm{opt}}, \underline{\theta}_{1}^{\mathrm{opt}}\right)=(0.366,1.004,6.377)$ for $\mathbf{u}_{1}$ and $\underline{\mathbf{s}}_{2}^{\mathrm{opt}}=$ $\left(\underline{\gamma}_{2}^{\text {opt }}, \underline{\lambda}_{2}^{\text {opt }}, \underline{\theta}_{2}^{\text {opt }}\right)=(0.107,1.814,21.362)$ for $\mathbf{u}_{2}$.

For $\mathbf{u}_{\mathbf{1}}$ and $\mathbf{u}_{2}$, figure 7 shows the experimental observations $\left\{L_{1}^{\exp }\left(\omega ; \eta_{j}\right)\right\}_{j}$ and $\left\{L_{2}^{\exp }\left(\omega ; \eta_{j}\right)\right\}_{j}$ from the reference model, together with their respective mean value estimates $m_{L_{1}^{\text {exp }}}$ and $m_{L_{2}^{\text {exp }}}$ and the output from the updated mean model with $\underline{\mathbf{s}}_{1}^{\text {opt }}$ and $\underline{\mathbf{s}}_{2}^{\text {opt }}$. Figure 7 shows that the updated mean model yields an excellent prediction with respect to the reference model. However it should be noted that for $\mathbf{u}=\mathbf{u}_{\mathbf{2}}$ the reference model exhibits a high level dispersion around the frequency 80 
Hz. These variabilities will be taken into account by the probabilistic model in the section below.

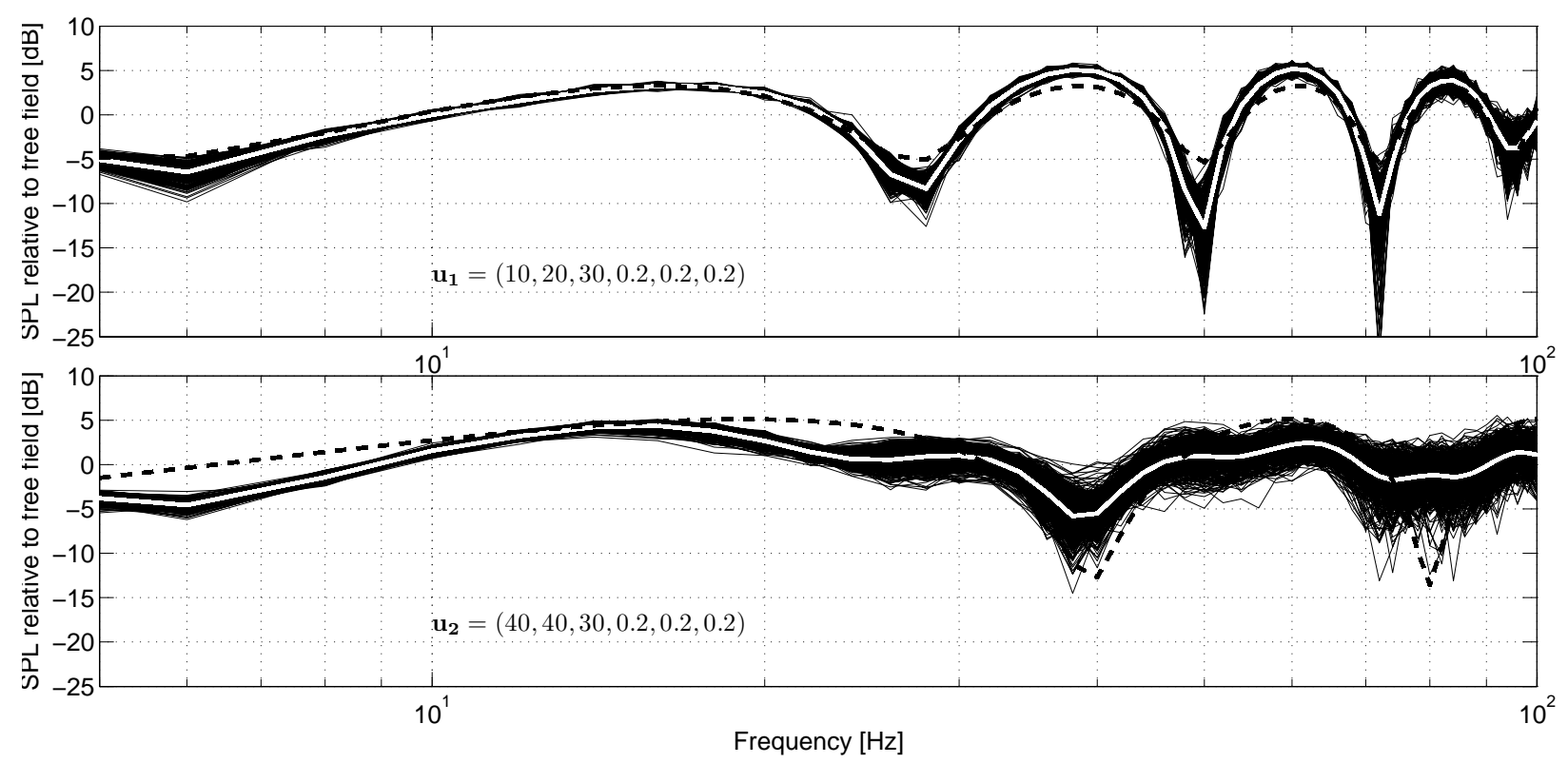

Figure 7: Comparisons of the reference model with the updated mean model. Reference model (made up of 500 outputs (thin lines) and the mean values estimates $m_{L_{1}^{\exp }}$ and $m_{L_{2}^{\text {exp }}}$ (thick white lines)). Updated mean model output $\underline{L}^{\underline{L}^{\text {opt }}}$ (dashed line). The value of parameter $\mathbf{u}$ is $\mathbf{u}_{1}$ (top figure) and $\mathbf{u}_{2}$ (bottom figure).

\subsubsection{Identification of the optimal parameter of the stochastic model with a genetic algorithm}

The updated parameters of the mean model $\underline{\mathbf{s}}_{1}^{\text {opt }}$ and $\underline{\mathbf{s}}_{2}^{\text {opt }}$ are then used to define the reduced admissible set $\mathcal{W}^{\text {red }}$ of $\mathbf{w}$, which is used to initialise the genetic algorithm. For the evaluation of the mean-square norm the $0 \mathrm{~Hz}$ point is removed from the calculation and the probability level used for the construction of the confidence region is $P_{c}=0.98$. For the statistical reduction of information performed in the maximum likelihood method the order of decomposition $q$ (see equation (39)) is $q=12$. With this order of decomposition, the accuracy $\epsilon$ in equation (43) is such that $\epsilon<10^{-3}$, meaning that at least $99.9 \%$ of the information is contained within the random variables $\left\{Y_{1}(\mathbf{w}), \ldots, Y_{12}(\mathbf{w})\right\}$.

Concerning the genetic algorithm, the number of individuals in the population is set to $n_{i}=$ 50, for each individual $v=500$ observations from the stochastic model are generated and the population evolved over 50 generations. At each generation, half the population is replaced with children. 
Figure 8 shows the values of the objective functions $-\mathcal{L}^{\text {red }}(\mathbf{w})$ and $J(\mathbf{w})$ of the 50 individuals at generation 1, 10 and 50 (the final generation) for both parameters $\mathbf{u}_{1}$ and $\mathbf{u}_{2}$. It can be seen that as the population evolves the values of the objective functions $-\mathcal{L}^{\text {red }}(\mathbf{w})$ and $J(\mathbf{w})$ decreases.

The chosen criteria to select the final individuals is the mean-square norm: individuals that show the lowest mean-square norm are selected first and within the remaining individuals, the individual that exhibit the highest log-likelihood is selected. This way, the least possible underestimation of the experimental observations is achieved. The corresponding optimal parameters $\mathbf{w}_{1}^{\text {opt }}$ and $\mathbf{w}_{2}^{\text {opt }}$ are

$$
\mathbf{w}_{1}^{\mathrm{opt}}=\left(m_{\Gamma}^{\mathrm{opt}}, m_{\Lambda}^{\mathrm{opt}}, m_{\Theta}^{\mathrm{opt}}, \sigma_{\Gamma}^{\mathrm{opt}}, \sigma_{\Lambda}^{\mathrm{opt}}, \sigma_{\Theta}^{\mathrm{opt}}\right)=(0.213,0.984,5.136,0.140,0.023,0.143)
$$

and

$$
\mathbf{w}_{2}^{\mathrm{opt}}=\left(m_{\Gamma}^{\mathrm{opt}}, m_{\Lambda}^{\mathrm{opt}}, m_{\Theta}^{\mathrm{opt}}, \sigma_{\Gamma}^{\mathrm{opt}}, \sigma_{\Lambda}^{\mathrm{opt}}, \sigma_{\Theta}^{\mathrm{opt}}\right)=(0.262,1.776,20.575,0.132,0.091,5.036)
$$
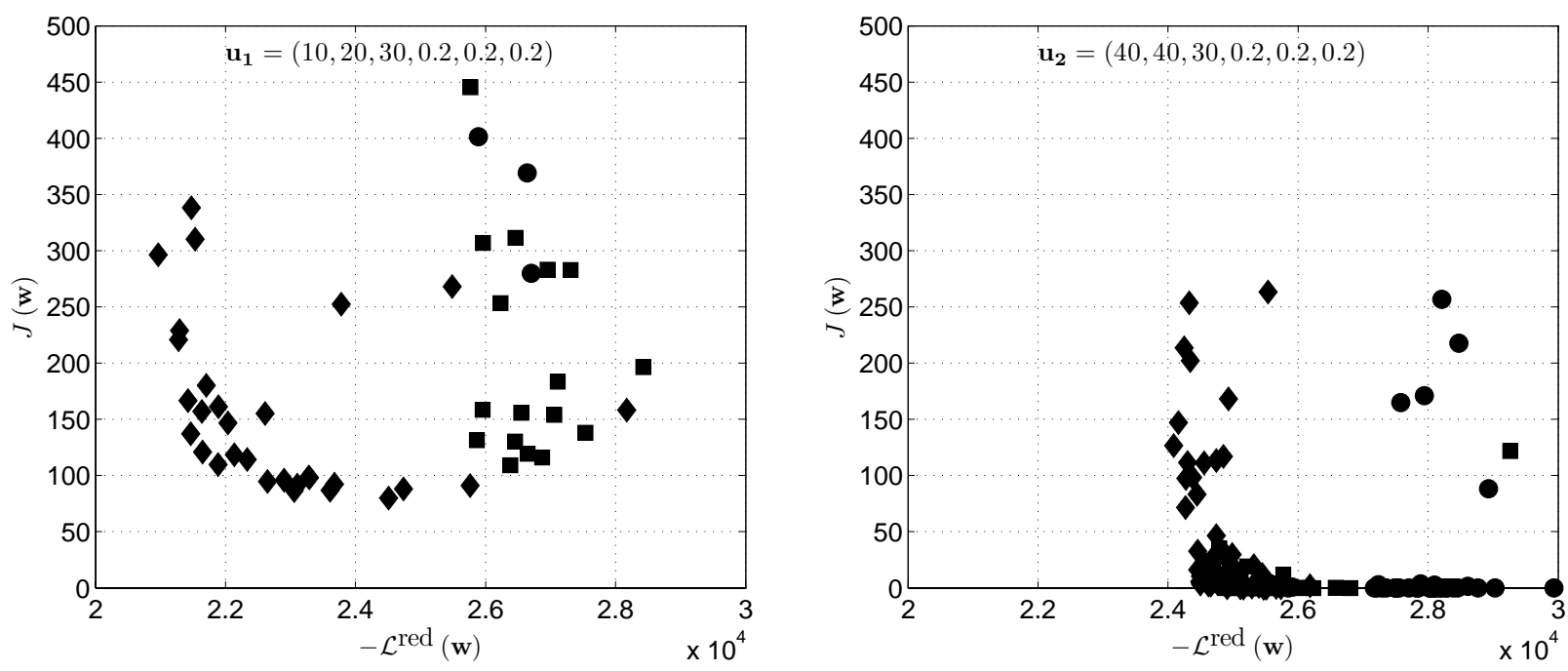

Figure 8: Values of the objective functions $-\mathcal{L}^{\text {red }}(\mathbf{w})$ and $J(\mathbf{w})$ for the 50 individuals at generations 1 (circles), 10 (squares) and 50 (final generation, diamonds). The value of parameter $\mathbf{u}$ is $\mathbf{u}_{\mathbf{1}}$ (left figure) and $\mathbf{u}_{\mathbf{2}}$ (right figure). Note that some individuals are out of the figure ranges. 


\subsection{Solution of the stochastic propagation model, construction of confidence region and valida-}

tion

Once the parameters $\mathbf{w}_{1}^{\text {opt }}$ and $\mathbf{w}_{2}^{\text {opt }}$ are identified for $\mathbf{u}_{\mathbf{1}}$ and $\mathbf{u}_{2}$ one can solve the stochastic equations (24) and (25) using the Monte Carlo method with these parameters and construct the associated confidence region as explained in section 8.3. Confidence regions are constructed with a probability level $P_{c}=0.98$ and with $v=500$ realisations of the stochastic model.

Figure 9 shows the experimental observations $\left\{L_{1}^{\exp }\left(\omega ; \eta_{j}\right)\right\}_{j}$ and $\left\{L_{2}^{\exp }\left(\omega ; \eta_{j}\right)\right\}_{j}$, their mean values estimates and the confidence region calculated using the stochastic model and the mean value of the stochastic model. As one can see the experimental observations belong to the constructed confidence regions. For configuration corresponding to $\mathbf{u}_{2}$, the solutions calculated using the stochastic model show a broad confidence region in the very low frequency range, whereas the reference solutions exhibit a thin confidence region. This means that the underlying deterministic model used is not robust in this very low frequency range with respect to statistical fluctuations generated by the probabilistic model.

\section{Conclusions}

In this work the development of a stochastic model for long-range non-linear sound propagation over urban environments has been initiated. It provides an insight into the construction method of stochastic models and demonstrates the feasibility of using such methods for studying sound propagation in complex environments. Furthermore, it is shown that the amount of information provided by such prediction models can be improved by the use of stochastic methods.

The mean propagation model is in this paper based on the non-linear parabolic equation and its extension for propagation over porous ground layers in order to model propagation over urban environment. This mean model exhibit low numerical cost but in counterpart induces model uncertainties for simulation of sound propagation over urban environments. Indeed the high complexity of the urban environment requires more advanced models. The mean propagation model is hence improved introducing a probabilistic model of uncertainties.

The constructed stochastic model was shown to be in good agreement with the reference model and can thus be used to study non-linear wave propagation in complex environments. Dissipation 


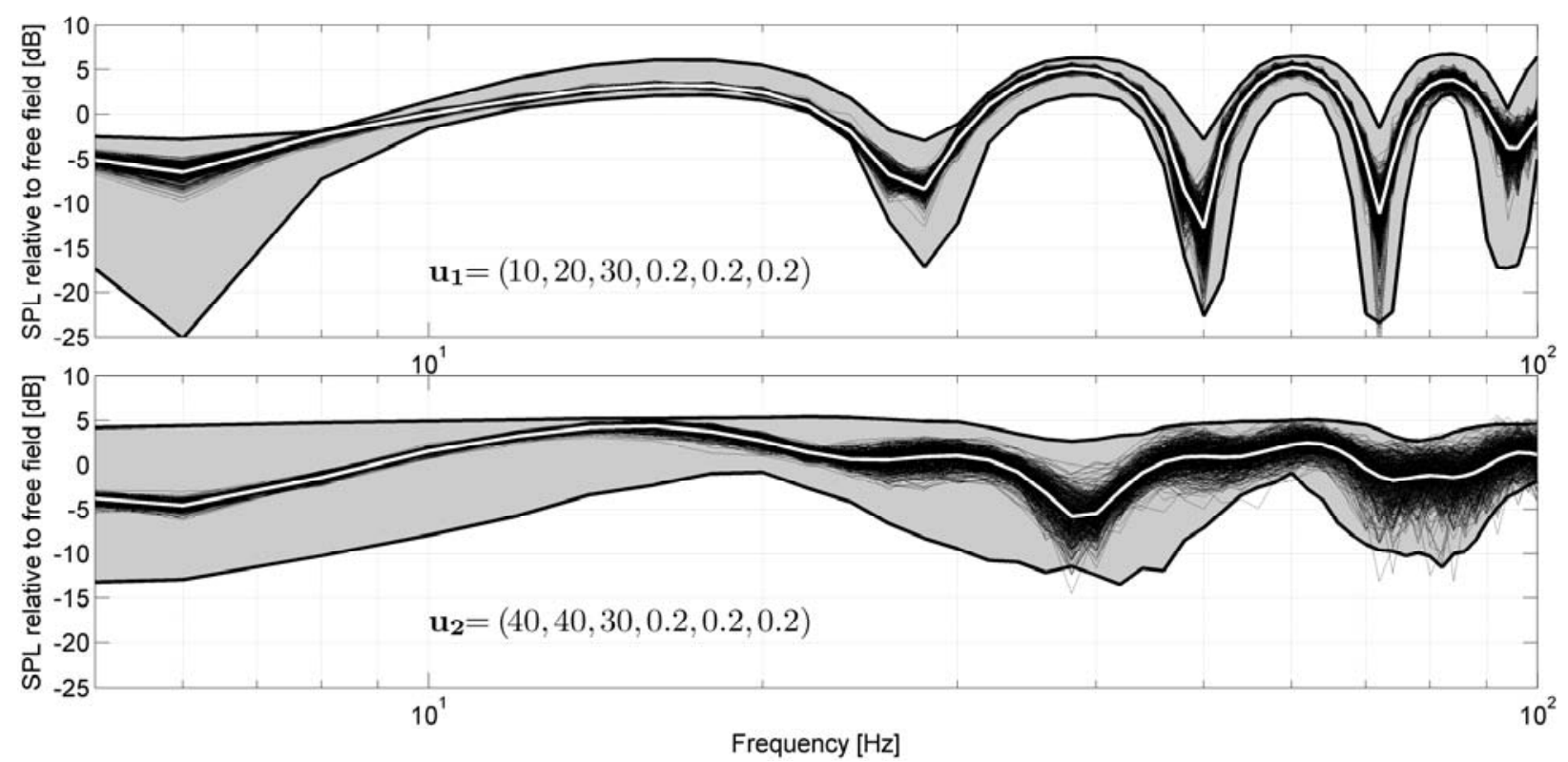

Figure 9: Comparisons of the reference model with the confidence region calculated with the stochastic model. Reference model (made up of 500 outputs (thin lines) and the mean values estimates $m_{L_{1}^{\exp }}$ and $m_{L_{2}^{\exp }}$ (thick white lines)). The confidence region calculated with the stochastic model is represented by the grey area, delimited by thick black lines. The value of parameter $\mathbf{u}$ is $\mathbf{u}_{\mathbf{1}}$ (top figure) and $\mathbf{u}_{\mathbf{2}}$ (bottom figure). 
effects or refraction effects are naturally present in the NPE model and could be incorporated in the stochastic model. Thanks to the low numerical effort associated with this model, large parametric studies could be performed, including main features of sound propagation outdoors, e.g. temperature and wind velocity gradients or site topography.

\section{References}

[1] J. J. Ambrosiano, D. R. Plante, B. E. McDonald, and W. A. Kuperman. Nonlinear propagation in an ocean acoustic waveguide. J. Acoust. Soc. Am., 87:1473-1481, 1990.

[2] K. Attenborough, A. Cummings, P. Dutta, P. Schomer, E. Salomons, E. Standley, O. Umnova, F. van den Berg, F. van der Eerden, P. van der Weele, and E. Védy. Blast sound absorbing surfaces. Technical report, ERDC/CRREL, September 2004.

[3] J. P. Bérenger. A perfectly matched layer for the absorption of electromagnetic waves. J. Comp. Phys., 114:185200, 1994.

[4] P. Blanc-Benon, L. Dallois, and D. Juvé. Long range sound propagation in a turbulent atmosphere within the parabolic approximation. Acust. Acta Acust., 87:659-669, 2001.

[5] J. P. Boris and D. L. Book. Flux-corrected transport III. minimal error FCT algorithms. J. Comp. Phys., 20:397$431,1976$.

[6] P. Caine and M. West. A tutorial on the nonlinear progressive wave equation (NPE) - Part 2. derivation of the three dimensional cartesian version without use of perturbation expansions. Appl. Acoust., 45:155-165, 1995.

[7] K. Castor, P. Gerstoft, P. Roux, and W. A. Kuperman. Long-range propagation of finite-amplitude acoustic waves in an ocean acoustic waveguide. J. Acoust. Soc. Am., pages 2004-2010, 2004.

[8] R. D. Ciskowski and C. A. Brebbia. Boundary Element Methods in Acoustics. Elsevier Applied Science, London, 1991.

[9] F. Collino. Perfectly matched absorbing layers for the paraxial equations. J. Comp. Phys., 131:164-180, 1997.

[10] K. Deb, A. Pratap, S. Agarwal, and T. Meyarivan. A fast elitist multiobjective genetic algorithm: NSGA-II. IEEE Transactions on Evolutionary Computation, 6:182-197, 2002.

[11] J. Defrance, E. Salomons, I. Noordhoek, D. Heimann, B. Plovsing, G. Watts, H. Jonasson, X. Zhang, E. Premat, I. Schmich, F. Aballea, M. Baulac, and F. de Roo. Outdoor sound propagation reference model developed in the European Harmonoise project. Acust. Acta Acust., 93:213-227, 2007.

[12] M. H. DeGroot. Probability and Statistics (2nd ed.). Addison-Wesley, London, 1980.

[13] J. E. Dennis. Nonlinear Least-Squares, State of the Art in Numerical Analysis, pages 269-312. Academic Press, 1977. 
[14] F. R. DiNapoli and R. L. Deavenport. Theoretical and numerical Green's function field solution in a plane multilayered medium. J. Acoust. Soc. Am., 67:92-105, 1980.

[15] K. E. Gilbert and M. J. White. Application of the parabolic equation to sound propagation in refracting atmosphere. J. Acoust. Soc. Am., 85:630-637, 1989.

[16] J. M. Hammersley and D. C. Handscomb. Monte Carlo Methods. Chapman and Hall, 1964.

[17] E. T. Jaynes. Information theory and statistical mechanics. Physical Review, 106 and 108:620-630 and 171-190, 1957.

[18] P. Jean. A variational approach for the study of outdoor sound propagation and application to railway noise. $J$. Sound and Vib., 212:275-294, 1998.

[19] I. T. Jolliffe. Principal Component Analysis. Springer-Verlag, New York, 1986.

[20] J. Kragh, B. Plovsing, S. Storeheier, G. Taraldsen, and HG. Jonasson. Nordic environmental noise prediction methods, summary report, Denmark: Delta, av 1719/01, 2002.

[21] T. Leissing. Nonlinear outdoor sound propagation - A numerical implementation and study using the nonlinear progressive wave equation. Master's thesis, Chalmers University of Technology, Göteborg, 2007.

[22] T. Leissing. Nonlinear wave propagation in complex media-Application to propagation over urban environements. PhD thesis, Université Paris-Est, 2009. Available at http://tel.archives-ouvertes.fr/tel-00455590/en/.

[23] T. Leissing, J. Jean, J. Defrance, and C. Soize. Nonlinear parabolic equation model for finite-amplitude sound propagation over porous ground layers. J. Acoust. Soc. Am., 126:572-581, 2009.

[24] T. Leissing, P. Jean, J. Defrance, and C. Soize. Nonlinear parabolic equation model for finite-amplitude sound propagation in an inhomogeneous medium over a non-flat, finite-impedance ground surface. In J. Acoust. Soc. Am., volume 123, page 3570, Paris, France, June 29 - July 4 2008. Acoustics'08, Second ASA (Acoustical Society of America) - EAA (European Acoustics Association) joint international conference.

[25] T. Leissing, P. Jean, J. Defrance, and C. Soize. Nonlinear parabolic equation model for finite-amplitude sound propagation over porous ground layers. pages 50-60, Ecole Centrale de Lyon, Ecully, France, 16-17 October 2008. 13th Long Range Sound Propagation Symposium (LRSPS).

[26] B. E. McDonald. High-angle formulation for the nonlinear progressive wave equation model. Wave Motion, 31:165-171, 2000.

[27] B. E. McDonald, P. Caine, and M. West. A tutorial on the nonlinear progressive wave equation (NPE) - Part 1. Appl. Acoust., 43:159-167, 1994.

[28] B. E. McDonald and W. A. Kuperman. Time domain formulation for pulse propagation including nonlinear behaviour at a caustic. J. Acoust. Soc. Am., 81:1406-1417, 1987.

[29] W. Press, B. Flannery, S. Teukolsky, and W. Vetterling, editors. Numerical recipes in Fortran, chapter 2, pages 42-43. Cambridge University Press, Cambridge, 1996.

[30] W. Press, B. Flannery, S. Teukolsky, and W. Vetterling, editors. Numerical recipes in Fortran, chapter 10, pages 
395-398. Cambridge University Press, Cambridge, 1996.

[31] R. Raspet, S. W. Lee, E. Kuester, D. C. Chang, W. F. Richards, R. Gilbert, and N. Bong. A fast-field program for sound propagation in a layered atmosphere above an impedance ground. J. Acoust. Soc. Am., 77:345-352, 1985.

[32] R. J. Serfling. Approximation Theorems of Mathematical Statistics. John Wiley and Sons, Hoboken, New Jersey, 1980.

[33] C. E. Shannon. A mathematical theory of communication. Bell System Tech. J., 1948. 27, 379-423 and 623-659.

[34] C. Soize. Random matrix theory for modeling uncertainties in computational mechanics. Comput. Methods Appl. Mech. Engrg., 194:1333-1366, 2005.

[35] C. Soize, E. Capiez-Lernout, J. F. Durand, C. Fernandez, and L. Gagliardini. Probabilistic model identification of uncertainties in computational models for dynamical systems and experimental validation. Comput. Methods Appl. Mech. Engrg., 198:150-163, 2008.

[36] J. C. Spall. Introduction to Stochastic Search and Optimization. John Wiley and Sons, Hoboken, New Jersey, 2003.

[37] N. Srinivas and K. Deb. Multiobjective optimization using nondominated sorting in genetic algorithms. Evolutionary Computation, 2:221-248, 1994.

[38] G. P. Too and J. H. Ginsberg. Cylindrical and spherical coordinate versions of the NPE for transient and steadystate sound beams. J. Vib. Acoust., 114:420-424, 1992.

[39] G. P. J. Too and S. T. Lee. Thermoviscous effects on transient and steady-state sound beams using nonlinear progressive wave equation models. J. Acoust. Soc. Am., 97:867-874, 1995.

[40] F. van der Eerden and E. Védy. Propagation of shock waves from source to receiver. Noise Cont. Eng. J., 53:87-93, 2005.

[41] D. van Maercke and J. Defrance. Development of an analytical model for outdoor sound propagation within the harmonoise project. Acust. Acta Acust., 93:201-212, 2007.

[42] E. Védy. Simulations of flows in porous media with a flux corrected transport algorithm. Noise Cont. Eng. J., 50:211-217, 2002.

[43] E. Walter and L. Pronzato. Identification of Parametric Models from Experimental Data. Springer, 1997. 